\title{
Unicast based reactive \& proactive routing simulation in MANET: A comparative study for protocols in mobility scenarios
}

\author{
Saber Hameed Yadgar ${ }^{1}$, Murad Ghazy Khalaf Alabdullah ${ }^{2}$, Bassam Mohsin Atiyah ${ }^{3}$, Kaesar Sabah \\ Khalaf $^{4}$ \\ ${ }^{1}$ Department of Electronic Techniques, Kirkuk Technical Institute, Northern Technical University, Iraq \\ ${ }^{2}$ Department of Electronic Techniques, Kirkuk Technical Institute, Northern Technical University, Iraq \\ ${ }^{3}$ Department of Electronic Techniques, Kirkuk Technical Institute, Northern Technical University, Iraq \\ ${ }^{4}$ Department of Electronic and Control Engineering, Kirkuk Technical College, Northern Technical University, Iraq
}

\begin{abstract}
A challenging issue for Ad-Hoc network is finding an effective routing mechanism, the wireless connection must have the capability to interact while traveling randomly. Although MANETs are self-configuring mobile node networks, the mobility of the nodes imposes some limitations on the power capacity of the nodes and thus on the transmission ranges. Many studies have investigated the protocols of Ad-Hoc networks, but this study has made a comprehensive investigation by using different scenarios among the studied protocols, particularly (DSR), (AODV) and (DSDV). The different numbers of deployed nodes with a mobile target node in the network have been analyzed with various important metrics such as; Generated, sent and received packet, lost and discarded packets, throughput, and the end to end latency with different packet sizes. Simulations are carried out in the simulation environment (NS2), and several comparisons were made among different metrics and scenarios by using different utilities. The results obtained showed interesting findings particularly when the scenario became more complex and the number of nodes increased. The number of packets received decreases by the mobile target node with the increasing number of nodes in the network. AODV showed better efficiency than the DSR and table-based DSDV protocol especially when a large number of nodes deployed. Furthermore, the average of the received packets to the sent packet ratio decreases proportionally as the number of nodes increases while the average end-to-end delay increases with the increasing number of nodes.
\end{abstract}

Keywords: $\quad$ MANET; Routing Protocols; Throughput; End-To-End Delay

\section{Corresponding Author:}

Murad Ghazy Khalaf Alabdullah

Department of Electronic Techniques, Northern Technical University

Kirkuk Technical Institute, Kirkuk, Iraq

Email: murad.kerkukly@gmail.com

\section{Introduction}

MANET is a set of mobile nodes with infrastructure-free networks. These nodes generate temporary networks without the help of centralized or standard devices that are regularly supported on traditional networks [2], [3]. In MANET, broadcasting is done in four different ways: Broadcast, Unicast, Multicast, and Anycast. In Broadcast, the packet sent by the sender is received by the recipients in the entire network. In Unicast, packets are sent independently by the sender to each receiver. In Multicast, packets sent by the sender at one time are copied over the network and delivered to each recipient. The routing protocol performs an important role if 
the two computers or nodes want to change the packet, and especially if a direct connection is not an available option [8]. These nodes find the way and are involved in the maintenance of the routes of other existing network nodes. If a node is added into a network, the situation becomes more complex. For the routing mechanism, a decision must be made for the best path between nodes [12]. To ensure proper routing, minimizing bandwidth is needed. The main characteristic that distinguishes hassle-free networks from other moving architectures is that the node movement causes a continuous reconstruction of the network topology [1]. Continuous topology changes in unpredictable networks cause overload. If this overload is not prevented, the network's resources will become exhausted and worthless. The problem is to balance the two. Overload; the use of bandwidth is measured by the power consumption and the processing needs of the mobile nodes [19]. Creating a strategy that effectively balances these conflicting needs forms the basis of the challenge [4]. Although conventional routing protocols can be used in the ad-hoc networks, each moving node acts as a router. Nevertheless, there are some problems with this approach:

- The transfer between the two nodes does not need to operate in both directions on the wireless network.

- Many "connections" between routers can be redundant by the routing algorithm.

- Network bandwidth is wasted when sending routing updates periodically.

- Battery power is wasted while sending routing updates periodically.

- Traditional routing protocols are not designed for dynamic topology change types that may exist in an Adhoc network.

The purpose of this research is to assess important protocols proposed for wireless networks of (Ad-hoc) based on performance evaluation by using different metrics. DSDV, DSR, and AODV routing protocols evaluation were done by simulation in the NS-2 simulation environment.

\section{Related works}

Many scientists attempt to discover the optimal protocol that can be implemented to a specified network and they used distinct situations to get the highest outcomes, this paper also evolves some of the previous studies in this area.

1) Neeraj Verma \& Sarita Soni [1] summarized advantage and disadvantages, characteristics and features of MANET, they have argued that MANET routing protocols are defined on the basis of the area and state of operation and that it is not feasible to design a single protocol suitable for all MANETs.

2) Charu Wahi \& Sanjay Kumar Sonbhadra [2] presented a comparative study on DSDV, DSR, AODV, they concluded that in all cases, a single routing protocol can't fully perform best. Therefore, the routing protocol selection should be made carefully according to the specific application requirements.

3) Bharathi [3] made a comparison between AODV, DSR, TORA, Proactive, Reactive and Hybrid routing protocols, he investigated best performing protocol under different parameters like; latency, scalability, congestion, security, routing overheads, multiple routes \& routing schemes, his study revealed that developing a reliable ad hoc routing protocol is a challenging research issue against existing security solutions.

4) Bharti Kukreja \& Sanjeev Kambhra [5] proposed and compared the results of Ad hoc On-Demand Distance Vector (AODV) and Ad-hoc On-Demand Multipath Distance Vector Routing (AOMDV), the two major on-demand reactive routing protocols for MANETs. The quality assessments are measured by different simulation times, they proposed that the AOMDV is a better on-demand routing protocol than AODV when network load increases as it provides better packet transmission and throughput statistics. If the delay time is a problem, however, then the AODV routing protocol is recommended. 
5) Ubarhande [7] conducted a comparative study with distinct metrics using AODV and DSR (NS2) program. Research has shown that DSR shows good results in terms of packet loss, while AODV shows stronger efficiency than DSR when there is an enormous effort to send TCP packets.

6) Amer O. Abu Salem et al. [9] evaluated the performance of DSR depending on the study of two factors; cache size and simulation speed; the delivery ratio, end-to-end delay, and throughput were studied. Depending on different cache sizes and speeds, they showed DSR has a reasonable performance in terms of data packet transmission ratio, latency and end-to-end delay at speeds below $15 \mathrm{~m} / \mathrm{s}$, and the cache size did not play a critical role in network performance. While the cache size played a remarkable function at higher speeds; the greater the size of the cache, the greater the end-toend latency.

7) Charu Wahi \& Sanjay Kumar [10] performed an assessment-based comparison between two reactive routing protocols for AODV \& DSR. they proposed that the protocol should be chosen according to the particular implementation and setting due to the different constraints of the routing protocol.

8) Diaa Eldein Mustafa \& Othman O. Khalifa [18] studied and made a comprehensive classification for some of MANETs routing protocols, found that AODV, OLSR and TORA from the Flat-routing approach are powerful, highly adaptive, efficient and scalable distributed routing algorithm. Those protocols are efficient and adaptable for different application specifically real time applications such as video streaming or video conferencing.

9) Rohit Kumar [13] investigated five different protocols based on multiple models of mobility, he studied reactive and proactive routing protocols which are (AODV, DSR, OLSR, DSDV, and TORA). his parameters depended on packet delivery rate, average end-to-end latency, throughput, and overhead of routing. He suggested that DSR should perform well in high mobile dynamic network topology and that AODV should share the same.

10) S. Mohapatraa \& P.Kanungob [20] have studied and proved that the DSR provides a better packet delivery ratio than other protocols for high mobility conditions of nodes, making it appropriate for fast-moving random networks. Likewise, for network size assessment, if the network size is below $600 \times 600$ sqm, the DSR protocols outperform other routing protocols. If the size of the network reaches 600x600sqm, and if the primary criteria are PDR and throughput, the OLSR protocol is the best solution for the network that has high mobility.

11) Jagdale et al. [22] compared the protocols AODV and DSDV; they established that Distance Vector protocol is not really suitable for Ad hoc network. DSDV requires bandwidth because it periodically streams routing information, while AODV does not need to maintain routing table, resulting in lower overhead and bandwidth consumption and the throughput is stable in AODV since it does not require any route information to be maintained.

12) Neeraj Kumar Pandey et al. [23] reviewed and evaluated various routing protocols and their advantages and disadvantages to help to select the best protocol to satisfy all communication requirements such as network performance, power consumption, security, and reliability.

13) Muhammad Kashif Nazir et al. [25] examined various techniques for managing traffic management, security problems, different types of threats, routing protocols and challenges faced by MANET. They provided an overview of various security problems, attacks on physical, data, and network layers, and security solutions to these issues. In their paper, numerous routing protocols have been introduced also, but they proposed that still there are many open research questions that will demonstrate the best behavior in which circumstances.

\section{Unicast routing protocols classification}

Classification can be divided into non-uniform and uniform. Non-uniform orientation approaches are related to hierarchical network models to ease node structure and management [13], [15], [16]. 
In the uniform routing protocol, all mobile nodes play a similar function, importance, and functionality. Neither of the nodes in the routing plan plays a distinct role. Each node sends and replies control messages for routing on the same path [14], [21]. There are three categories of uniform routing protocols [17], [21]; these classes are proactive, hybrid, and reactive [6], [11]. The following protocols are emphasized in our research; proactive (DSDV), reactive (DSR) and (AODV) protocols.

\subsection{Definition of protocols}

\subsubsection{Destination-sequenced distance vector routing protocol (DSDV)}

This protocol sends its table to other nodes. If the change is large the whole table is sent, and if its small, only the changing part is sent. When there is an adjustment in the path to a node, the adjacent nodes are informed with a higher sequence number. Each node that receives this change updates the routing table and reports the change to its neighbors. In this way, the whole network becomes aware of this change. Updates are transmitted periodically or immediately after any topology change. The DSDV is a forward-looking protocol. Each node must have the information necessary to maintain its own routing table and each node knows the whole network as a graph [8].

\subsubsection{Dynamic source routing protocol (DSR)}

Network nodes cooperate to send packets to each other within multiple jumps between nodes, allowing communication via wireless transmission from one to another. The wireless communication conditions, like nodes moving in the network, joining the network or dropping the network, and interference of resources, the DSR protocol determines and automatically performs all routings. To achieve any goal, the number or order of the medium may change [9].

Each sent data packet carries the list of the order in which the nodes should pass on the header. The transmission route for each data can be inserted to the header of the packet, and when using it to forward it to other nodes, it can easily store this routing information in the future. In wireless networks, DSR allows connections to be used as directional connections when needed and overall performance improves network connectivity [9].

\subsubsection{Ad-hoc on-demand distance vector routing protocol (AODV)}

(AODV) is a demand-based algorithm. That is, if only needed by the source nodes, they construct routes between the nodes and these routes are only kept as long as they are necessary. It is actually a merger of DSR and DSDV. The DSR provides the optional route discovery and route maintenance basic mechanism from the DSDV to hop by hop. AODV always uses target sequence numbers to guarantee loop freedom, and when the converged network topology changes, it provides rapid convergence to avoid the problem of Bellman-Ford. Nodes that are not on available active routes do not provide routing data and do not include any regular changes to the routing table [18]. Furthermore, when dealing with the local connectivity of the mobile node, it may be aware of the other nodes around each moving node, using many techniques including local broadcasts. The neighboring node routing tables are arranged for the best use of response times to local movements, and for quick response times for corporate requests of new routes. The main objectives of the algorithm are: to publish packet discovery only when needed, to separate local connectivity methods (local detection) and general topology care, possibly to disseminate information about changes in the local connectivity of mobile neighboring nodes that require information [5].

\subsection{Comparison of protocols}

Qualitative analysis of the routing protocol can be performed in different criteria, Table 1 summarizes a comparison of unicast routing protocols for DSDV, DSR, and AODV protocols. 
Table 1. Comparison of protocols [2], [3]

\begin{tabular}{|c|c|c|c|}
\hline Protocol name & DSDV & DSR & AODV \\
\hline Routing protocol parameter & Proactive & Reactive & Reactive \\
\hline routes & Single & Multiple & Multiple \\
\hline Source route & None & Has & Has \\
\hline Route selection & Connection status & Shortcut & Shortcut \\
\hline Loop & None & None & None \\
\hline Target update & Neighbors & Source & Source \\
\hline Security & None & None & None \\
\hline Power saving & None & None & None \\
\hline Periodical broadcasting & Has & None & Has \\
\hline Control Traffic & High & Low & Low \\
\hline Delay & Low & High & High \\
\hline Routing information & Stored in table & Does not store & Does not store \\
\hline
\end{tabular}

\section{Simulation setup and performance results}

The routing in unicast protocols was executed in the NS2 network simulator; the simulation was conducted to assess the presented protocols. Various parameters were tested. The Table 2 contains all parameters used for the simulations.

Table 2. Parameters of simulation

\begin{tabular}{|c|c|}
\hline Parameter & Value \\
\hline Channel type & Channel/Wireless Channel \\
\hline Network interface type & Wireless \\
\hline MAC type & Mac/802_11 \\
\hline Interface queue max. length & $6 / 10 / 20 / 50 / 100$ \\
\hline Number of nodes & DSDV/DSR/AODV \\
\hline Routing protocol & $1200 \mathrm{x} 1200$ \\
\hline Size of the simulation area & $256,512,1024$ \\
\hline Packet size & 120 Seconds \\
\hline Simulation time & Random \\
\hline Topology & \\
\hline
\end{tabular}

The creation of a 6-node scenario using (NS 2) program takes 42 seconds, and it takes 5 minutes to create a 100-node scenario. The results of the Trace-graph program vary between 30 minutes and 3 hours depending on the scenario of the simulation. This study looked at the behavior of reactive AODV, DSR, and proactive DSDV routing protocols with settings and parameters for each protocol given in the network simulator table.

\subsection{Results comparison in terms of no. of nodes and packet size}

The performance of DSR, AODV, and DSDV is evaluated by taking (number of simulated nodes) parameters. The nodes are set to $6,10,20,50$, and 100 in the simulation. The position of the nodes is in a certain position throughout the simulation and only the target node simulation moves within a certain time. Although the target node is mobile, it keeps receiving packets sent from the initial source by using protocols. As with other simulations, the target, the number of packets received during the simulation, the detailed network information in the simulation and the rate of packets sent and received from the nodes are graphically displayed. Furthermore, the results are compared when the size of a packet is changed. 
4.2. Mobile target node with packet size $(256,512,1024)$

The performances of protocols were evaluated for various nodes setup and packet sizes in the simulations. Tables (3), (4) and (5) show all details about 20 and 50 nodes as a sample for the sent and received packets, discarded or thrown packets, throughput and end to end average latency if the DSDV, DSR, and AODV protocols are applied.

Table 3. DSDV protocol (mobile target node and packet size 256, 512 and 1024)

\begin{tabular}{|c|c|c|c|c|c|c|}
\hline \multirow{2}{*}{ Parameter } & \multicolumn{6}{|c|}{ Number of nodes } \\
\cline { 2 - 7 } & $\mathbf{2 0}$ & $\mathbf{5 0}$ & $\mathbf{2 0}$ & $\mathbf{5 0}$ & 20 & $\mathbf{5 0}$ \\
\hline Packet size & \multicolumn{2}{|c|}{256} & \multicolumn{2}{|c|}{512} & \multicolumn{2}{|c|}{1024} \\
\hline Total generated packets & $\mathbf{7 2 8 0 5}$ & $\mathbf{3 9 0 9 9}$ & $\mathbf{4 5 9 5 3}$ & $\mathbf{2 9 2 9 4}$ & $\mathbf{2 9 0 2 1}$ & $\mathbf{1 9 3 4 1}$ \\
\hline Sent packets & $\mathbf{1 1 3 1 9}$ & $\mathbf{6 3 6 2}$ & $\mathbf{9 5 1 0}$ & $\mathbf{5 4 8 9}$ & $\mathbf{7 0 2 9}$ & $\mathbf{3 7 4 1}$ \\
\hline Lost packets & $\mathbf{0}$ & $\mathbf{4 3 8 6}$ & $\mathbf{1 1 3 4 8}$ & $\mathbf{1 6 5 3}$ & $\mathbf{4 2 9 4}$ & $\mathbf{0}$ \\
\hline Discarded packets & $\mathbf{3 4 7 4 4}$ & $\mathbf{1 0 0 7 3}$ & $\mathbf{1 0 7 5 0}$ & $\mathbf{9 7 9 9}$ & $\mathbf{1 0 9 6 0}$ & $\mathbf{9 8 5 5}$ \\
\hline Received packets & $\mathbf{2 5 1 9}$ & $\mathbf{1 6 2 1}$ & $\mathbf{1 7 4 1}$ & $\mathbf{1 4 0 4}$ & $\mathbf{1 1 3 7}$ & $\mathbf{8 5 8}$ \\
\hline Throughput & $\mathbf{0 , 2 2 2}$ & $\mathbf{0 , 2 5}$ & $\mathbf{0 , 1 8 3}$ & $\mathbf{0 , 2 5 5}$ & $\mathbf{0 , 1 6 1}$ & $\mathbf{0 , 2 2}$ \\
\hline End-to-end average latency & $\mathbf{0 , 1 0 5}$ & $\mathbf{0 , 1 9}$ & $\mathbf{0 , 2 9 3}$ & $\mathbf{0 , 3 1 6}$ & $\mathbf{0 , 3 3 6}$ & $\mathbf{0 , 4 9 2}$ \\
\hline
\end{tabular}

Table 4. DSR protocol (mobile target node and packet size 256, 512 and 1024)

\begin{tabular}{|c|c|c|c|c|c|c|}
\hline \multirow{2}{*}{ Parameter } & \multicolumn{6}{|c|}{ Number of nodes } \\
\hline & 20 & 50 & 20 & 50 & 20 & 50 \\
\hline Packet size & \multicolumn{2}{|c|}{256} & \multicolumn{2}{|c|}{512} & \multicolumn{2}{|c|}{1024} \\
\hline Total generated packets & 128997 & 147101 & 84259 & 104991 & 60525 & 72052 \\
\hline Sent packets & 21205 & 20531 & 15498 & 16894 & 11989 & 11429 \\
\hline Lost packets & 36169 & 37805 & 21202 & 26670 & 12671 & 14315 \\
\hline Discarded packets & 12324 & 13598 & 11307 & 14091 & 11484 & 14830 \\
\hline Received packets & 5257 & 4839 & 3755 & 3002 & 1966 & 1575 \\
\hline Throughput & 0,247 & 0,255 & 0,242 & 0,177 & 0,163 & $\mathbf{0 , 1 3 7}$ \\
\hline End-to-end average latency & 0,144 & 0,361 & 0,217 & 0,683 & 0,661 & 1,327 \\
\hline
\end{tabular}

Table 5. AODV protocol (mobile target node and packet size 256, 512 and 1024)

\begin{tabular}{|c|c|c|c|c|c|c|}
\hline \multirow{2}{*}{ Parameter } & \multicolumn{6}{|c|}{ Number of nodes } \\
\cline { 2 - 7 } & 20 & 50 & 20 & 50 & 20 & 50 \\
\hline Packet size & \multicolumn{2}{|c|}{256} & \multicolumn{2}{|c|}{512} & \multicolumn{2}{|c|}{1024} \\
\hline Total generated packets & 124467 & $\mathbf{1 3 7 6 6 4}$ & $\mathbf{8 3 8 3 7}$ & $\mathbf{9 6 4 8 5}$ & $\mathbf{5 4 5 2 2}$ & $\mathbf{5 8 2 1 8}$ \\
\hline Sent packets & $\mathbf{1 8 1 7 8}$ & $\mathbf{1 7 9 9 9}$ & $\mathbf{1 3 9 2 3}$ & $\mathbf{1 5 1 6 8}$ & $\mathbf{1 0 8 0 8}$ & $\mathbf{9 3 6 9}$ \\
\hline Lost packets & $\mathbf{3 5 1 9 4}$ & $\mathbf{3 7 4 0 2}$ & $\mathbf{2 2 8 1 3}$ & $\mathbf{2 5 0 3 1}$ & $\mathbf{1 3 9 2 5}$ & $\mathbf{1 1 3 6 3}$ \\
\hline Discarded packets & $\mathbf{1 1 8 4 1}$ & $\mathbf{2 7 4 0 2}$ & $\mathbf{1 1 7 9 6}$ & $\mathbf{2 5 4 1 8}$ & $\mathbf{1 1 7 0 2}$ & $\mathbf{2 6 6 1 7}$ \\
\hline Received packets & $\mathbf{5 6 4 2}$ & $\mathbf{4 2 9 6}$ & $\mathbf{3 3 6 9}$ & $\mathbf{3 3 9 4}$ & $\mathbf{1 8 2 5}$ & $\mathbf{1 5 8 8}$ \\
\hline Throughput & $\mathbf{0 , 3 1 0}$ & $\mathbf{0 , 2 3 8}$ & $\mathbf{0 , 2 4 1}$ & $\mathbf{0 , 2 2 3}$ & $\mathbf{0 , 1 6 8}$ & $\mathbf{0 , 1 2 0}$ \\
\hline End-to-end average latency & $\mathbf{0 , 1 4 3}$ & $\mathbf{0 , 1 7 9}$ & $\mathbf{0 , 1 4 5}$ & $\mathbf{0 , 1 7 9}$ & $\mathbf{0 , 2 7}$ & $\mathbf{0 , 4 4 2}$ \\
\hline
\end{tabular}

Figure 1 show noumber of packets sent when the DSDV, DSR and AODV protocol is used in the mobile target node state, as the number of nodes that are randomly separated increases in the mobile target node, the 
number of packets sent decreases in the DSDV protocol. The reason we get these different results is that; when the deployed nodes amount increases, the required time to prepare the routing table increases, if we increase the number of deployed nodes with the DSR or AODV protocol, the packets sent will increase as well, then it degrades gradually. As the routing protocols for DSR and AODV are the same (on-demand) routing protocols, it seems that the two have the same performance.

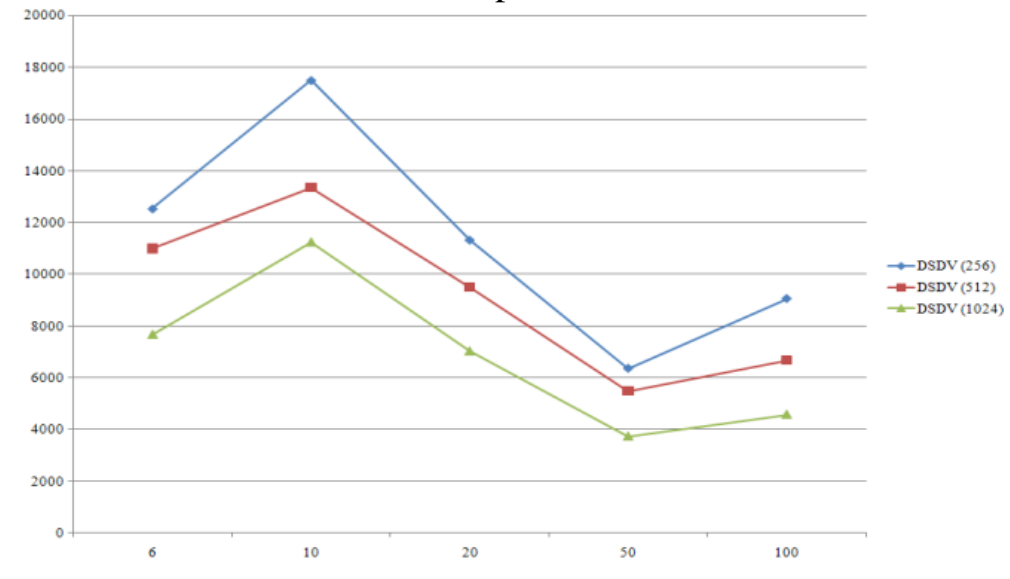

Fig. 1-a) (DSDV)

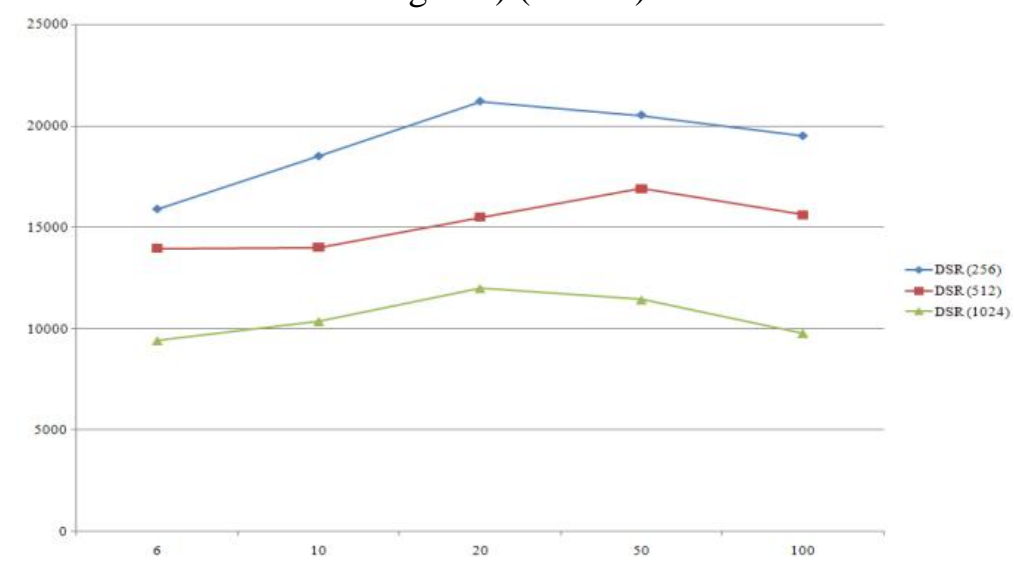

Fig. 1-b) (DSR)

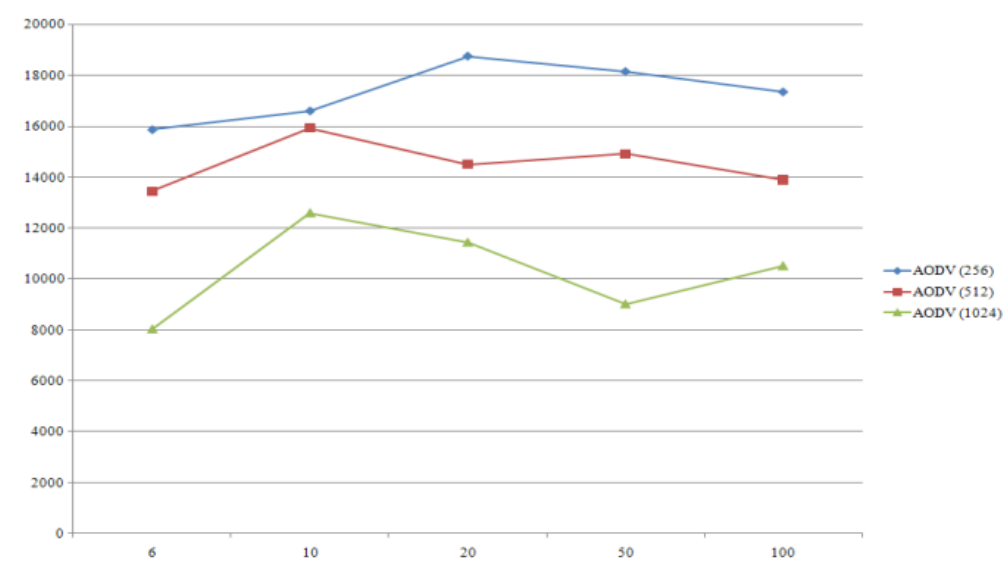

Fig. 1-c) (AODV)

Figure 1. Number of packets sent in every protocol in case of (mobile target node)

In all protocols regarding the number of packets received, the number of packets received decreases with the increasing number of nodes when the target node is mobile. This decrease may be due to increased network 
traffic and congestion. Figure 2 show the number of packets received when using the DSDV protocol in the case of a mobile target node.

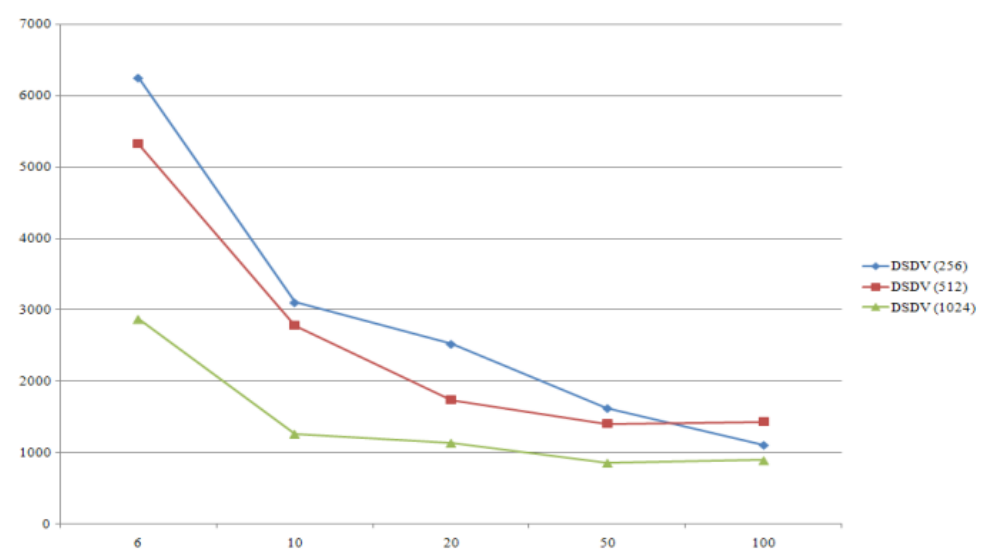

Fig. 2-a) (DSDV)

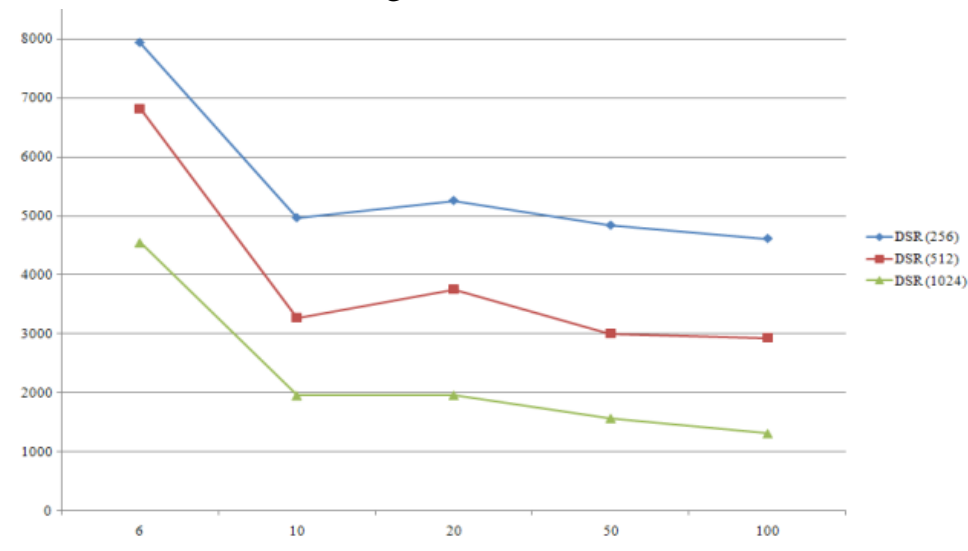

Fig. 2-b) (DSR)

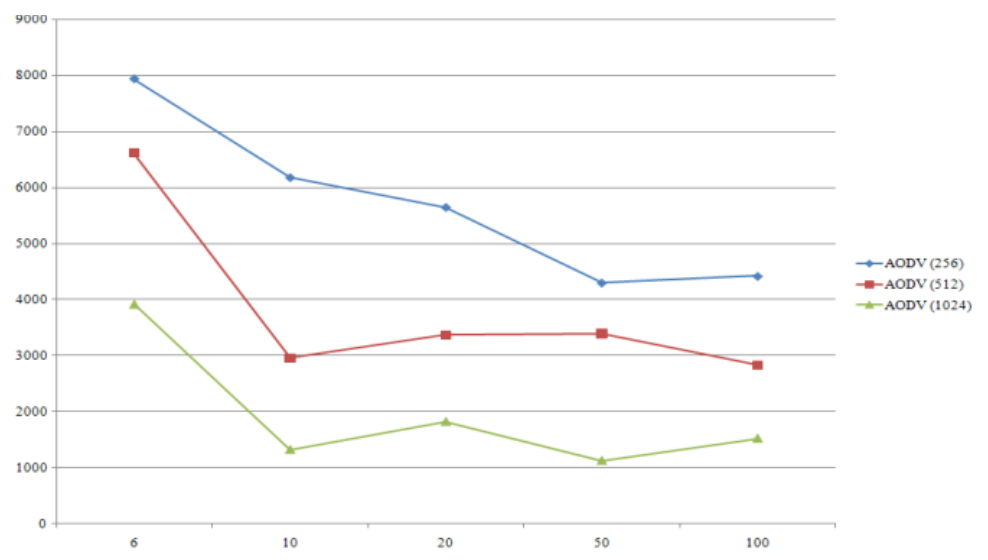

Fig. 2-c) (AODV)

Figure 2. Number of packets received in every protocol in case of (mobile target node)

The ratio is known as the average of the received packets to the sent packet. Generally, as the number of nodes increases in DSDV, DSR, and AODV, the ratio obtained decreases proportionally. Figure 3 show the ratio of mobile target node for each routing protocol. 


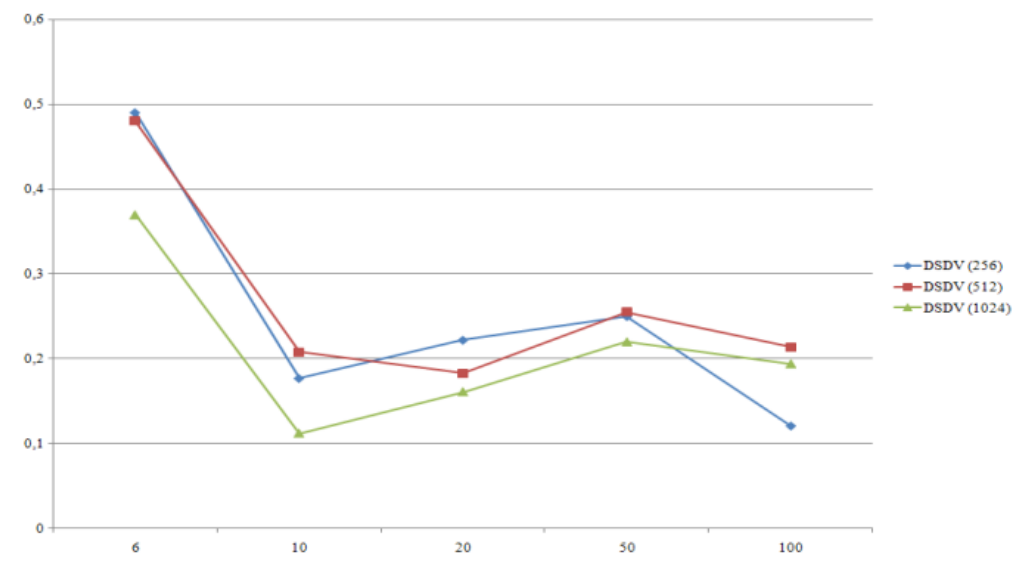

Fig. 3-a) (DSDV)

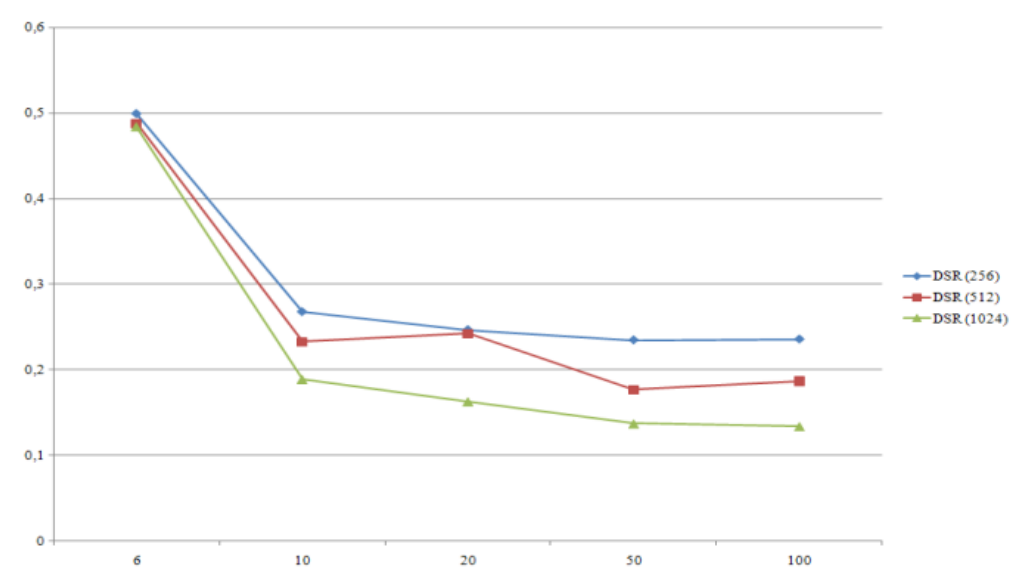

Fig. 3-b) (DSR)

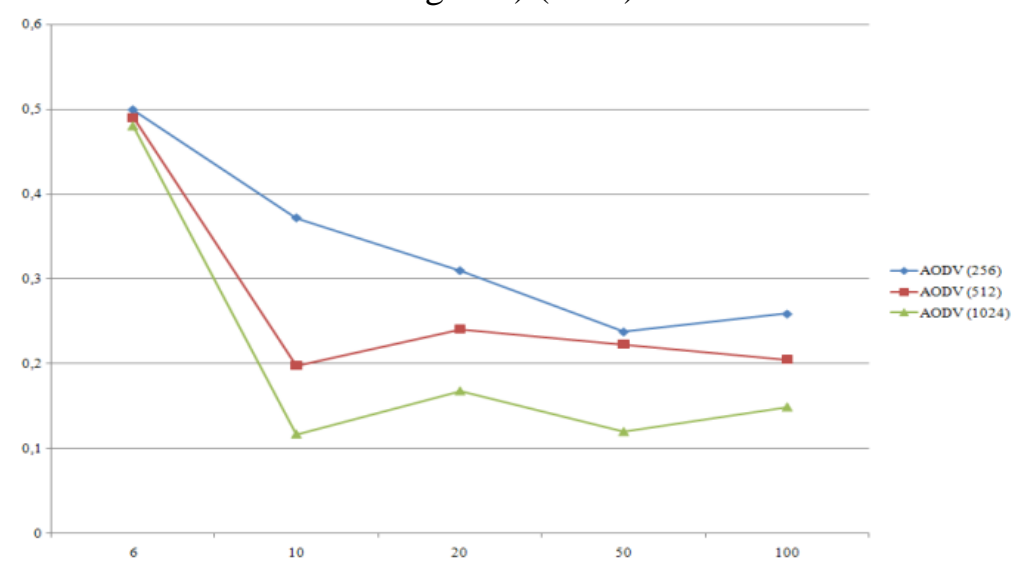

Fig. 3-c) AODV

Figure 3. Ratio obtained in every protocol in case of (mobile target node)

In the case of the mobile target node network, the average end-to-end delay increases with the increasing number of nodes when using DSDV, AODV, and DSR protocols with a packet size 256, 512 and 1024. Figure $(4 \mathrm{a}, 4 \mathrm{~b}$, and $4 \mathrm{c})$ show the end-to-end average latency when using these protocols. 


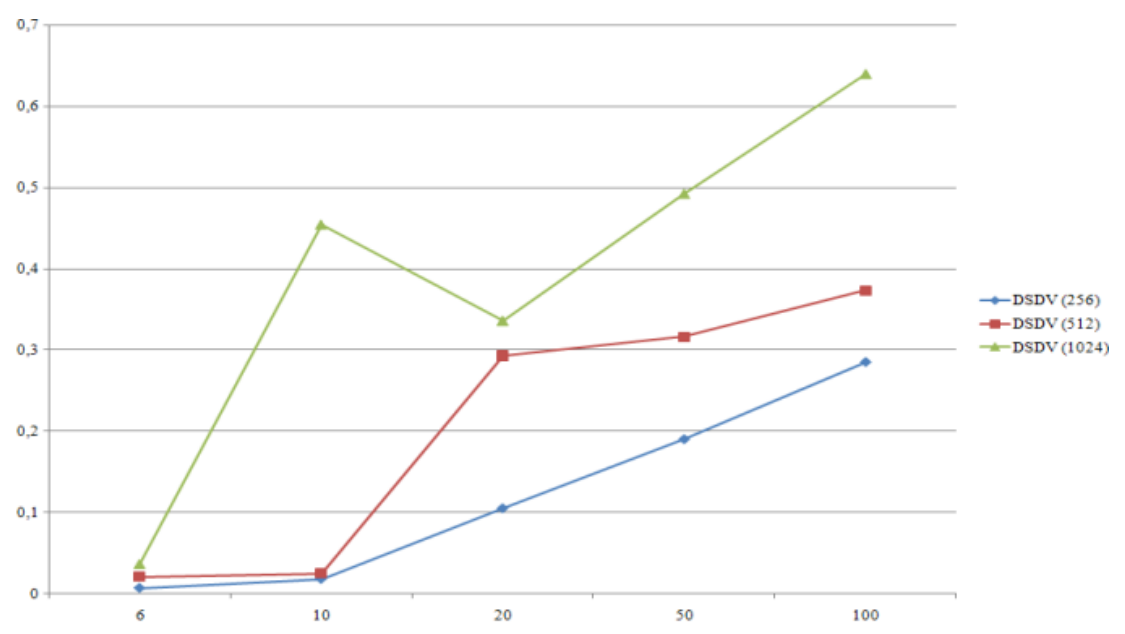

4-a) (DSDV)

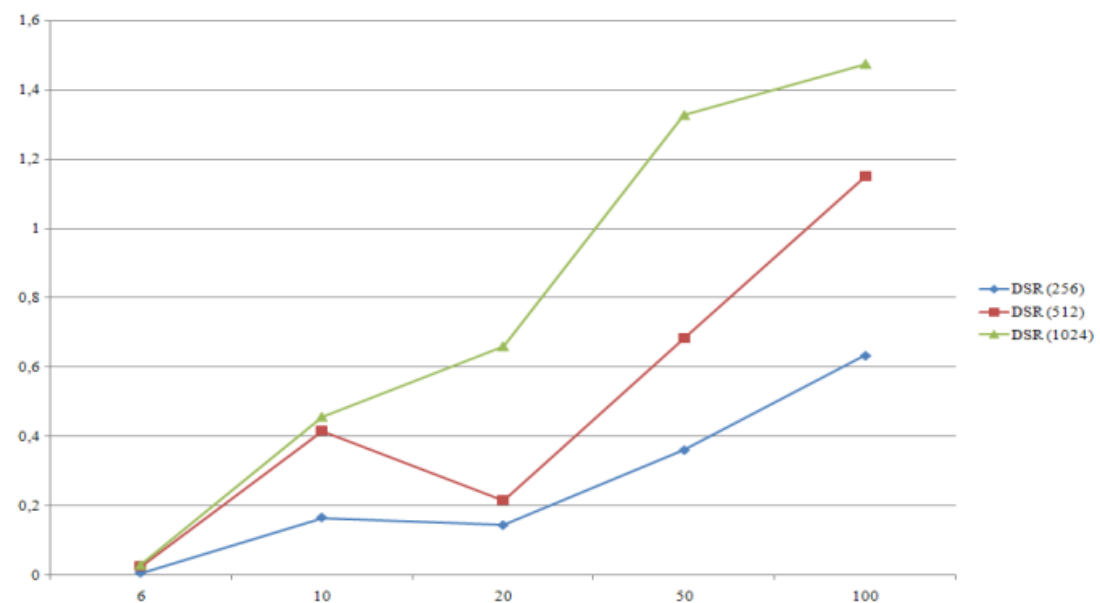

4-b) (DSR)

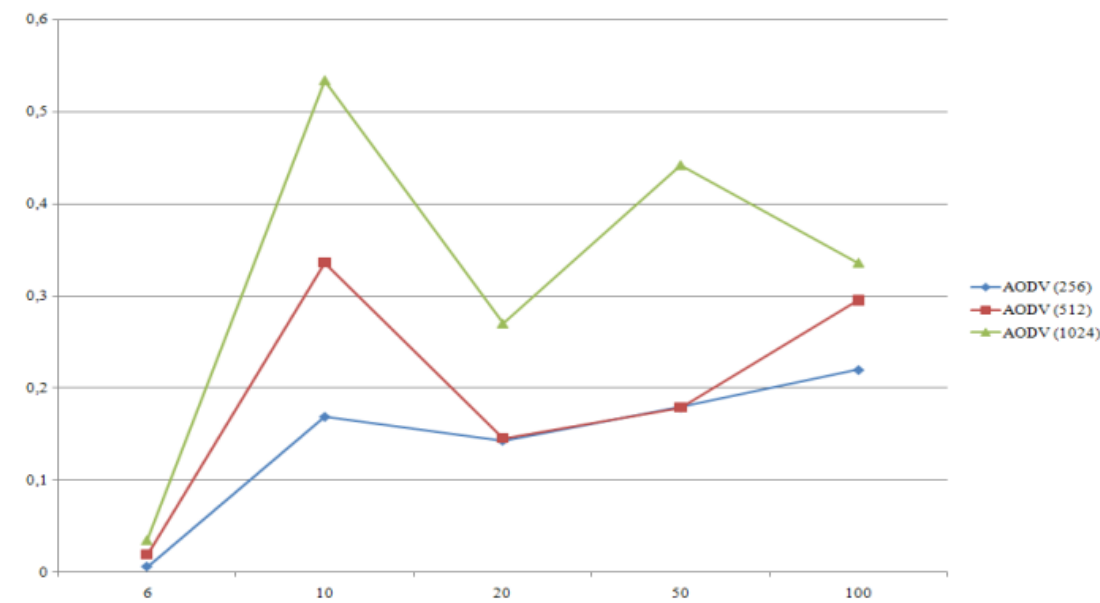

4-c) (AODV)

Figure 4. Average end-to-end delay in every protocol for the (mobile target node)

Figure 5 shows the 6-node ratio (average packets received) of the routing protocols in the target node moving state. Based on the 6-node results, the DSDV protocol performs less than DSR and AODV, but the DSR and AODV have the same performance. 


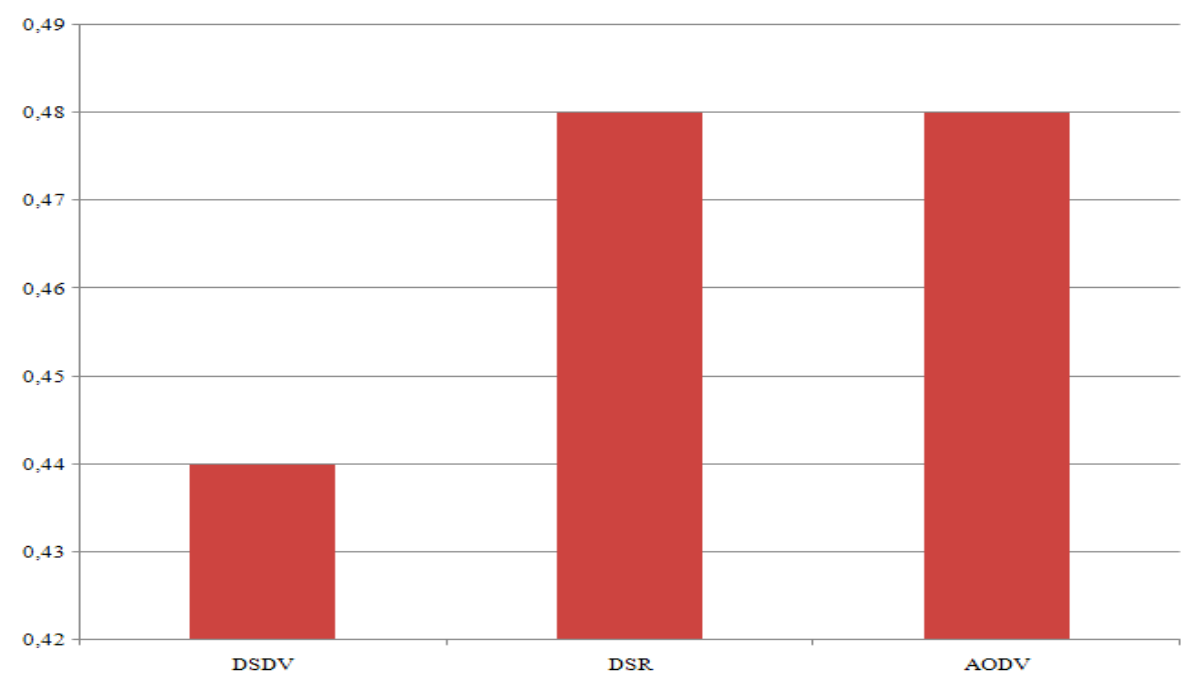

Figure 5. Average number of packets received for DSDV, DSR, and AODV for 6-node in the target node mobile state

On the other hand, for the AODV protocol, average packets for 100 nodes show better performance as shown in Figure 6 comparing to Figure 5 with 6 nodes deployment. Therefore, AODV protocol offers greater efficiency compared to the DSDV which demonstrates poor efficiency at 6 and 100 nodes.

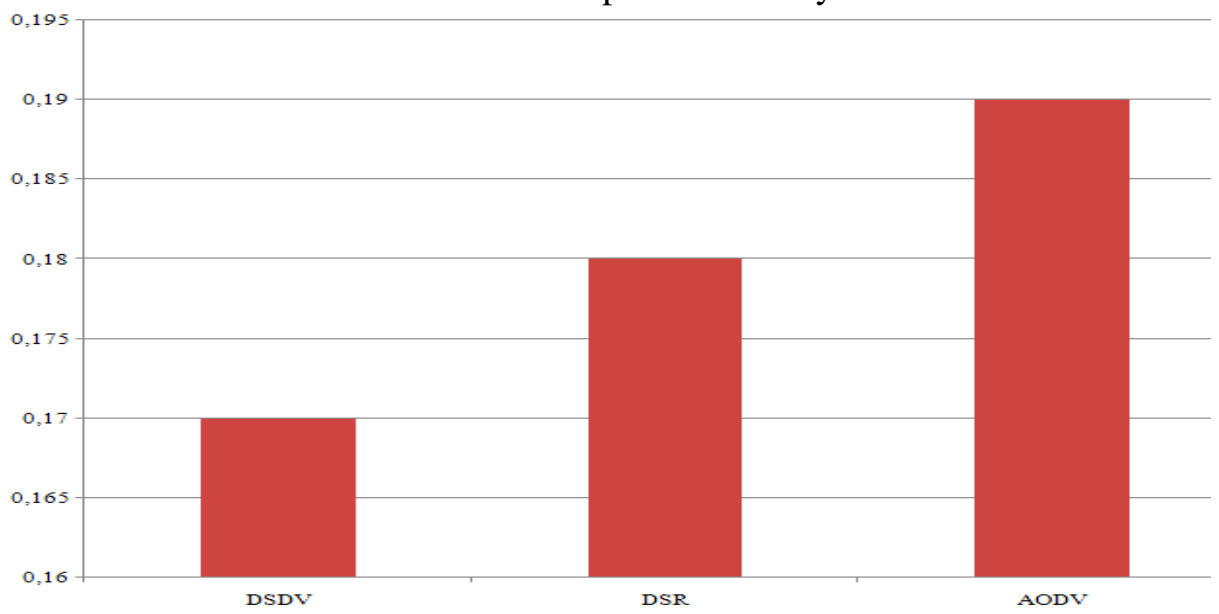

Figure 6. Average number of packets received for DSDV, DSR, and AODV for 100-node in the target node mobile state

\section{Conclusions}

An overview of the routing process for ad hoc networks is presented, and different metrics were compared for mobile target node in terms of end-to-end latency, packet delivering ratio subjected to the different numbers of packet sizes and nodes deployed in the network. Although many authors have studied and worked on the presented three MANET protocols, this article has investigated these protocols from another perspective with different scenarios, the study have used different methods and parameters to compare the results such as; the size of the area, simulation time, packet size, number of nodes.

The protocol overhead involved in updating all nodes with new routing information is much higher in DSDV than in AODV and DSR since routes in AODV and DSR are created only when they needed. The DSR uses the cache mechanism aggressively and stores multiple routes to the destination. AODV, on the other hand, uses the method of routing tables, destination sequence numbers, loops and determining whether or not the route exists, as a route to the destination. According to the simulation outputs, the DSDV indicates a decrease 
in the number of the packet sent and received as the number of nodes increases and the reason behind is that the DSDV protocol must notify the nodes on the network before sending the packet.

In addition, the average ratio obtained decreases proportionally as the number of nodes increases in all studied protocols. Hence, the packets received are greater in DSR and AODV protocols than the DSDV protocol. The number of packets received is generally greater when the target node is mobile. The reason is as follows, while the mobile target node passes through the network, the packet size will change, and the average of sent, lost, received, discarded, rate, and end-to-end latency are affected.

The average end-to-end delay of data packets consists of delays such as buffering during delayed route discovery, waiting in the interface queue, waiting for media access. The average end-to-end delay may increase. For route discovery in large networks, the delay in AODV is due to the number of control packets sent. As a result of this delay, packets waiting in the queue are discarded. However, the latency is less than DSDV and DSR. As a result, when the AODV protocol is used in a mobile target node, it performs better on both small networks and large networks. The DSR protocol performs better on the static network.

In summary, for packet loss, the DSDV protocol shown the best result, and the DSR is superior in generating packets, while in receiving packets DSR \& AODV were better. Nevertheless, because of differences in their design, the DSR and AODV protocols may present a variable performance for the different data packets and network sizes due to their algorithmic differences.

1. An increase in node numbers results in an increase in end-to-end average latency.

2. An increase in the size of the packet results in an increase in the mean latency end-to-end.

3. Compared to other protocols, when the nodes move continuously, AODV has the best performance.

4. DSR \& AODV is ideal for networks with low node and mobility speeds, whereas AODV is better for large networks. It has low latency and uses a smaller cache, making this ideal for low bandwidth and low power networks.

5. The DSDV shows that the number of packets sent and received decreases as the number of nodes increases.

As we address proactive and reactive routing protocols, the real thing that restrains the efficiency of the routing protocols is node mobility if node moves less or it might not move at all, because all the routing protocols work well when there is no mobility, but at the period of movement, there are several other things that are associated with the node like; traffic, communication quality, end-to-end latency.

\section{References}

[1] Neeraj Verma, Sarita Soni, "A Review of Different Routing Protocols in MANET", International Journal of Advanced Research in Computer Science, Vol. 8, No. 3, March - April 2017

[2] Charu Wahi, Sanjay Kumar Sonbhadra, "Mobile Ad Hoc Network Routing Protocols: A Comparative Study", International Journal of Ad hoc, Sensor \& Ubiquitous Computing (IJASUC), Vol.3, No.2, April 2012.

[3] Bharathi, "A Review and Comparative Study on Various Routing Protocols for MANET", International Journal of Innovative Research in Science, Engineering and Technology, Vol. 5, Issue 12, December 2016.

[4] Sachin Chauhan, "Performance Evaluation of Routing Protocols for Manet using NS2", International Journal of Computer Science and Mobile Computing, Vol. 4, No. 9, pg.242 - 249, 2015.

[5] Bharti Kukreja, Sanjeev Kambhra, "Performance Comparison of Routing Protocols in MANET", International Journal of Computer Science and Network 108 Security, Vol.14, No.8, August 2014.

[6] Anjali, \& Singh, M. "Simulation and Performance Analysis of AODV, OLSR, GRP Routing Protocols by considering IEEE 802.11 Standard", International Journal of Advanced Research in Computer Science and Software Engineering, Vol. 2, No. 6, pp.171-178, 2012. 
[7] Sachin Dnyandeo Ubarhande "Performance Evolution of AODV and DSR Routing Protocols in MANET Using NS2, International Journal of Scientific \& Engineering Research, Vol. 3, Issue 5, May-2012.

[8] Sarkar, "Routing Protocols", Ad Hoc Mobile Wireless Networks Principles Protocols and Applications, Second Edition, 2013.

[9] Amer O. Abu Salem, Ghassan Samara, Tareq Alhmiedat, "Performance Analysis of Dynamic Source Routing Protocol", Journal of Emerging Trends in Computing and Information Sciences, Vol. 5, No. 2 February 2014.

[10] Charu Wahi, Sanjay Kumar Sonbhadra, "Mobile Ad Hoc Network Routing Protocols: A Comparative Study", International Journal of Ad hoc, Sensor \& Ubiquitous Computing (IJASUC), Vol.3, No.2, April 2012.

[11] Ajay Kumar, Deepak Sharma, Sunil Kumar, Suneet Shukla, "Performance Valuation Of Reactive Routing protocols", International Journal of Research in Computer Applications and Robotics, Vol.1 Issue.6, Pg: 39-44 September 2013.

[12] Chander Prabha, Surender Kumar, Ravinder Khanna, "Wireless Multi-hop Ad-hoc Networks: A review", IOSR Journal of Computer Engineering (IOSR-JCE), Issue 2, Vol. 16, VI, pp54-62, 2014.

[13] Rohit Kumar, "Comparative Study of Reactive and Proactive Routing Protocols", International Journal of New Innovations in Engineering and Technology, Vol. 3 Issue 3 - July 2015.

[14] S.R. Raja, K. Alagarsamy, "Routing Protocols In Manet QoS Survey", International Journal of Innovative Research in Advanced Engineering (IJIRAE), Issue 5, Vol. 2, May 2015.

[15] Bharathi, "A Review and Comparative Study on Various Routing Protocols for MANET", International Journal of Innovative Research in Science, Engineering and Technology, Vol. 5, Issue 12, December 2016.

[16] Nariman Jabbar Qasim, et al. "Reactive protocols for unified user profiling for anomaly detection in mobile Ad Hoc networks", Periodicals of Engineering and Natural Sciences, Vol. 7, No. 2, pp.843-852, 2019.

[17] Hager Ramadan, et al. "Effective data routing using mobile sinks in disjoint mobile wireless sensor networks", Periodicals of Engineering and Natural Sciences, Vol. 7, No. 1, pp.82-98, 2019.

[18] Diaa Eldein Mustafa Ahmed, Othman O. Khalifa, "A Comprehensive Classification of MANETs Routing Protocols", International Journal of Computer Applications Technology and Research, Vol. 6, No. 3, 141-158, 2017.

[19] Arappali Nedumaran, V. Jeyalakshmi, "2- CAERP: A Congestion and Energy Aware Routing Protocol for Mobile Ad Hoc Network", Indian Journal of Science and Technology, Vol. 8, No. 35, 2015.

[20] S. Mohapatra, P.Kanungo, "Performance analysis of AODV, DSR, OLSR and DSDV Routing Protocols using NS2 Simulator", Procedia Engineering, Vol. 30, pp. 69 - 76, 2012.

[21] Murad Ghazy Khalaf, et. al. "Analysis and simulation of three MANET routing protocols: A research on AODV, DSR \& DSDV characteristics and their performance evaluation”, Periodicals of Engineering and Natural Sciences, Vol.7, No.3, pp.1228-1238, 2019.

[22] Prof. B.N. Jagdale, Prof. Pragati Patil, Prof. P. Lahane, Prof. D. Javale, "Analysis and Comparison of Distance Vector, DSDV and AODV Protocol of MANET", International Journal of Distributed and Parallel Systems (IJDPS), Vol.3, No.2, 2012.

[23] Neeraj Kumar Pandey, Amit Kumar Mishra, Pushpneel Verma, “A Comparative Study of MANET Routing Protocols”, Journal of Mobile Computing, Communications \& Mobile Networks, Vol.1, Issue 1, 2014.

[24] Dejan Tepsic, Mladen Veinovic, “ Comparative Analysis of Unicast Routing Protocols in Manet Networks", Applied informatics and education, International Scientific Conference of IT and BusinessRelated Research, Synthesis-115, 2015.

[25] Muhammad Kashif Nazir, Rameez U. Rehman, Atif Nazir, "A Novel Review on Security and Routing Protocols in MANET”, Communications and Network, Vol. 8, pp.205-218, 2016. 